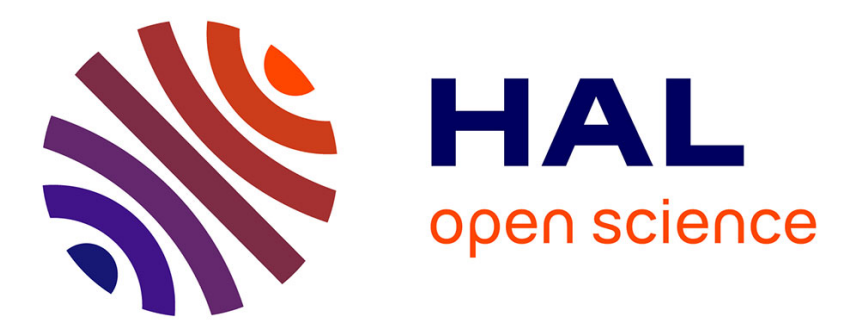

\title{
Les monnaies méditerranéennes en Gaule du Nord (150 av.-100 apr. J.-C.). L'apport des contextes archéologiques \\ Stéphane Martin
}

\section{- To cite this version:}

Stéphane Martin. Les monnaies méditerranéennes en Gaule du Nord (150 av.-100 apr. J.-C.). L'apport des contextes archéologiques. Revue Numismatique, 2013, 170, pp.329-354. 10.3406/numi.2013.3209 . hal-01278201

\section{HAL Id: hal-01278201 \\ https://hal.science/hal-01278201}

Submitted on 23 Feb 2016

HAL is a multi-disciplinary open access archive for the deposit and dissemination of scientific research documents, whether they are published or not. The documents may come from teaching and research institutions in France or abroad, or from public or private research centers.
L'archive ouverte pluridisciplinaire HAL, est destinée au dépôt et à la diffusion de documents scientifiques de niveau recherche, publiés ou non, émanant des établissements d'enseignement et de recherche français ou étrangers, des laboratoires publics ou privés.

\section{(ㅇ)(1) 80}

Distributed under a Creative Commons Attribution - NonCommercial - ShareAlikel 4.0 


\title{
Les monnaies méditerranéennes en Gaule du Nord (150 av.-100 apr. J.-C.). \\ L'apport des contextes archéologiques
}

\begin{abstract}
Résumé - La récente étude de J.-M. Doyen sur les monnaies d'Ebusus découvertes dans le nord de la Gaule permet d'affirmer, selon son auteur, que du numéraire méditerranéen en bronze circulait en tant que tel dès la première moitié du ier siècle av. J.-C., en dehors de toute influence romaine. L'étude des monnaies méditerranéennes découvertes en contexte archéologique dans la zone étudiée semble au contraire montrer que la conquête romaine joue un rôle déterminant pour la pénétration en Gaule septentrionale de ces pièces, quel que soit le métal. Un lien fort avec la présence militaire romaine consécutive à la guerre des Gaules apparaît probable.
\end{abstract}

Mots clés - Gaule du Nord, monnaies méditerranéennes, guerre des Gaules, romanisation, armée, commerce.

Summary - According to J.-M. Doyen's recent study on Ebusan coins found in northern Gaul as early as the first half of the $1^{\text {st }} \mathrm{c}$. BC, Mediterranean bronze coins were used as currency in this region, which lay outside Roman influence. However, the study of stratified finds makes it likely that the Roman conquest played a great part in bringing such coins (both in bronze and silver) to northern Gaul. They can be linked with the military presence in the region in the years following the Gallic Wars.

Keywords - Northern Gaul, Mediterranean coins, Gallic wars, Romanization, army, trade.

Le cas de la monnaie d'Ebusus découverte sur l'oppidum du Vieux-Laon, est maintenant bien connu ${ }^{1}$. Identifiée dans les abondantes récoltes de surface de ce site gaulois qui pourrait être le Bibrax césarien, cette monnaie est, pour B. Lambot et P. Casagrande, le témoin de la présence de frondeurs baléares sur le site, qu'on pouvait lier à un épisode précis de la guerre des Gaules. J.-M. Doyen, dans un article très récent sur les monnaies d'Ebusus, remet en cause l'interprétation proposée par B. Lambot, au profit de contacts commerciaux plus anciens entre la Gaule septentrionale et la péninsule ibérique. Il conclut : «Le dernier élément non négligeable est de montrer, pour la première fois en Gaule septentrionale,

* Doctorant, École pratique des hautes études - AnHiMA UMR 8210 / ATER, Université Toulouse II-Le Mirail. Courriel : martin.st@laposte.net.

1. Lambot, Casagrande 1997. 
que des monnaies de bronze grecques (au sens large) peuvent apparaître dans des contextes datables du LT D2 $\mathrm{a}^{2}$, comme petit numéraire à part entière, en dehors de toute influence romaine. ${ }^{3}{ }^{»}$

Le but de cette contribution n'est pas de contester l'hypothèse argumentée de J.-M. Doyen pour expliquer la diffusion des monnaies ébusitaines, qui privilégie l'idée d'un commerce atlantique indépendant des puissances méditerranéennes. Mais la phrase citée plus haut, de caractère général, appelait vérification. En effet, la question des influences méditerranéennes, et en particulier romaines, dans le développement des sociétés gauloises, est un des thèmes majeurs de la recherche actuelle. Même si on est maintenant loin de la fausse alternative «barbarie (gauloise) ou civilisation (gréco-romaine)», et que les évolutions endogènes des sociétés de la fin de l'âge du fer sont reconnues, le problème n'en est pas pour autant tranché.

Des études détaillées telles que celle de J.-M. Doyen manquent pour d'autres séries $^{4}$; en conséquence, la conclusion citée qui s'applique avant tout à une série particulière, risque d'être généralisée à la plupart des découvertes de monnaies méditerranéennes connues. C'est pourquoi il a semblé utile de proposer la liste de toutes les monnaies méditerranéennes, hors monnaies romaines, trouvées en Gaule du Nord dans des contextes archéologiques publiés et bien datés ${ }^{5}$. Nous espérons ainsi préciser la chronologie de l'arrivée de ces pièces, loin de leur zone de circulation traditionnelle. Les données pourront sembler peu nombreuses, voire peu significatives. Il nous semble pourtant indispensable de s'appuyer en priorité sur elles. En effet, seul le recours à des informations contextuelles précises permettra de poser des bases pour l'interprétation ; si étroites qu'elles risquent d'être, ces bases n'en seront pas moins solides et rigoureuses ${ }^{6}$.

La zone examinée comprend la totalité de la Gaule Belgique dans son extension augustéenne, ainsi que les civitates de Gaule Lyonnaise qui la bordent au sud (figure 1). La vallée du Rhône a n'a pas été prise en compte ; la pénétration des monnaies méridionales par le couloir rhodanien rend en effet la situation

2. Pour La Tène D2a, soit entre 90/80 et 60/50 av. J.-C.

3. DOYEN 2011, p. 283.

4. Hors de la zone méditerranéenne, les répertoires publiées de monnaies étrangères pour la Gaule sont rares et concernent essentiellement l'ouest de la France (HIERNARd 1982, BeYNEIX, Piot 1995, Santrot, Aubin 2002. Adam 2007 traite de l'ensemble de la Gaule, mais seulement pour les Ive et III siècle av. J.-C.). Le seul travail systématique en Gaule septentrionale couvre la Belgique actuelle : BAR 1991, 1996, 2002. Le travail déjà ancien de B. Fischer (1978) ne concerne que les monnaies originaires d'Afrique. On trouvera les données pour la Gaule du sud dans Feugère, Py 2011. À l'exception de ce dernier ouvrage, les mentions de contextes de découverte précis sont très rares.

5. Le dépouillement a été réalisé dans le cadre d'une thèse de doctorat dont la soutenance est prévue à l'automne 2013, sous la direction de M. Reddé et M. Amandry.

6. Une position adoptée, entre autres, par B. Lambot (2004, p. 123, n. 1). Pour des travaux prenant en compte le contexte stratigraphique des monnaies, voir par ex. HASELGROVE 1999 et 2005 (Gaule Belgique), Gruel, Popovitch 2007 (Bibracte) ou Luley 2008 (Lattes). 


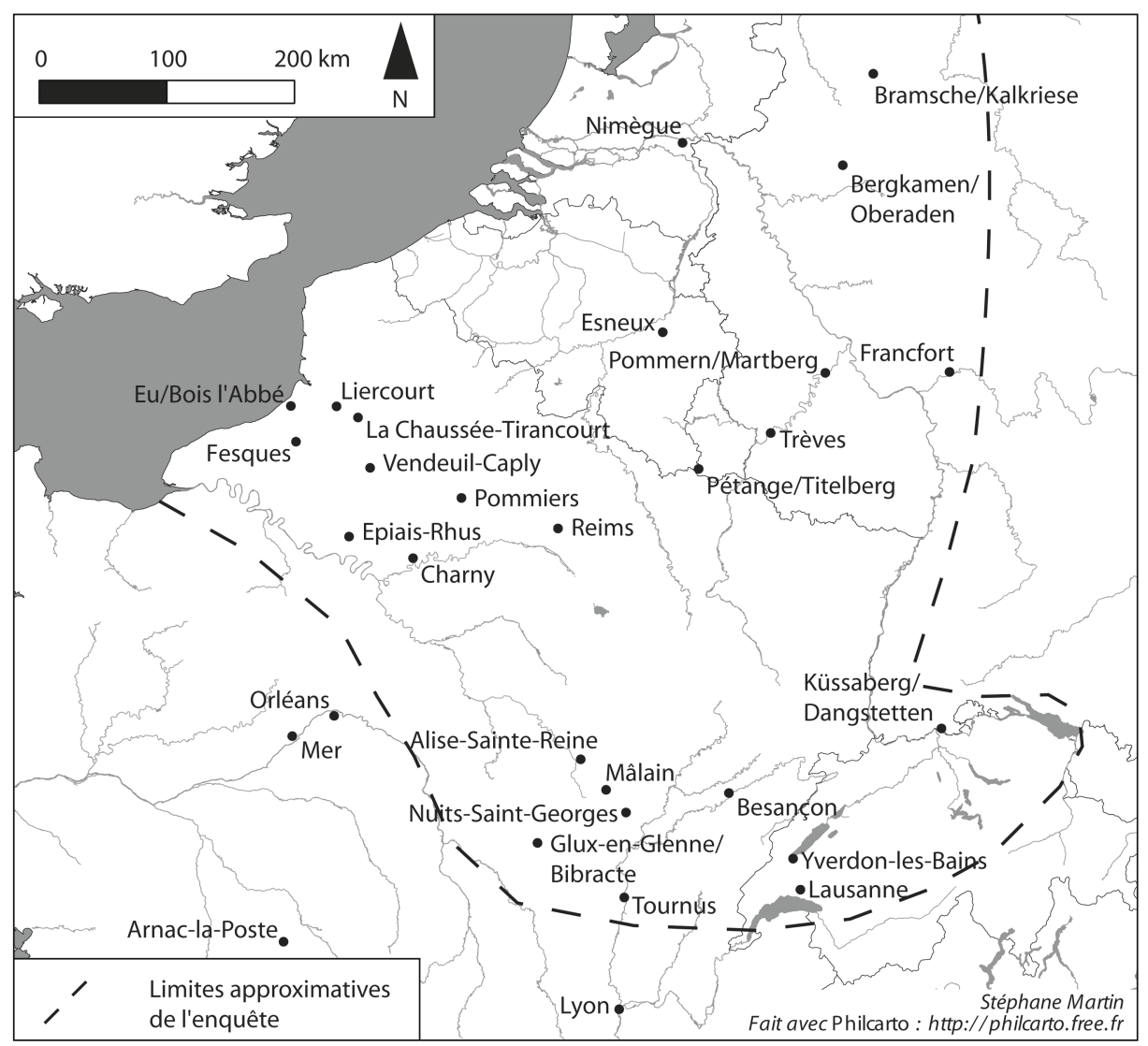

Figure 1 - Zone étudiée, avec sites mentionnés dans le texte.

complètement différente ${ }^{7}$. Les deux grands vides que l'on peut observer sur la carte (autour de la Belgique actuelle, et au nord-est de la France) sont d'abord dus à un déficit de contextes archéologiques publiés, et on se gardera de les interpréter autrement.

La recherche de contextes archéologiques a porté sur la période allant de 150 av. J.-C. à 100 apr. J.-C. Nous n'avons pas fait de recherches systématiques sur les contextes antérieurs, mais ils sont très peu nombreux, et ceux dont nous avons connaissance n'ont pas livré de monnaies «exotiques $»^{8}$. Ce fait nous semble

7. Comme nous l'a confirmé J. Genechesi, dont la thèse de doctorat porte sur la circulation monétaire pré-romaine dans la vallée du Rhône.

8. AdAM 2007, en particulier p. 258-259. Bien que l'article ne traite pas de monnaies, MILCENT 2006 rappelle les risques qui peuvent peser sur les découvertes anciennes de mobilier «exotique». 
d'ailleurs significatif ; l'absence de telles monnaies (par ailleurs connues seulement en petit nombre) dans les contextes archéologiques antérieurs au Ire siècle av. J.-C. montre une diffusion limitée et en aucun cas «systématique», qui les exclut, à notre sens, d'une circulation monétaire au sens classique du terme.

Nous avons pris en compte toutes les monnaies provenant du bassin méditerranéen, Italie, Gaule Transalpine, Marseille et vallée du Rhône incluses. Les monnaies romaines n'ont pas été répertoriées, pas plus que les provinciales romaines ${ }^{9}$. Au total, selon ces critères, nous avons trouvé 72 monnaies provenant de 23 sites, répartis dans une trentaine de contextes différents. On peut classer ces monnaies en huit groupes :

- Celles de Gaule méridionale, hors Marseille : il s'agit, outre deux monnaies à la croix, de petits bronzes du Languedoc (LT 2677, 2698, 4353), d'une monnaie en argent attribuée aux Rutènes (LT 3433) et d'un bronze ibéro-languedocien (LT 2403).

- Les oboles de Marseille en argent : la très grande majorité ont la tête à gauche ${ }^{10}$; ces séries semblent être frappées tardivement, même si certaines variantes semblent attestées dès le Ive siècle av. J.-C. On y a ajouté une obole qui présente des similarités avec les oboles archaïques, mais dont la provenance exacte reste inconnue (cat. 25).

- Les petits bronzes de Marseille : les exemplaires en contexte sont rares (deux seulement). Les deux sont identifiés comme étant du type LT 1673, mais il faut rappeler qu'il s'agit du seul petit bronze au taureau illustré dans le La Tour ; l'identification n'est donc pas à prendre littéralement.

- Les monnaies de la vallée du Rhône : il s'agit principalement de deniers au cavalier, la plupart appartenant au groupe IV, le plus tardif ${ }^{11}$.

- Les monnaies frappées en Italie : elles regroupent d'un côté des drachmes padanes, de l'autre de possibles petits bronzes pseudo-Ebusus/Massalia.

- La monnaie frappée en Illyrie : il s'agit d'un unique bronze frappé à Dyrrachium au IIe siècle av. J.-C.

- La monnaie frappée au Moyen Orient : très certainement la monnaie la plus ancienne du lot, un bronze à attribuer vraisemblablement à Antiochos III de Syrie (223-187 av. J.-C.).

- Les monnaies frappées en Afrique : il s'agit de deux deniers de Juba ${ }^{\mathrm{er}}$, vraisemblablement frappés dans les années 48-46 av. J.-C. sur un étalon romain.

9. La grande majorité est issue des ateliers ibériques, et on se reportera à ce sujet aux travaux de M.-P. García-Bellido (2004, en particulier). L'hypothèse militaire proposée par l'auteur est à nos yeux convaincante, bien que ses conclusions puissent être jugées trop détaillées.

10. Feugère, Py 2011, séries OBM-8 à OBM-11.

11. Dans le classement de Deroc 1983, qui reste valide même si la chronologie proposée a été révisée (voir en dernier lieu GENECHESI 2012). 
Les contextes sont étagés entre 120 av. J.-C. et 100/110 apr. J.-C., mais près des trois-quarts sont antérieurs à 20 apr. J.-C. Afin d'étudier les rythmes d'arrivée de ces monnaies «étrangères », nous avons groupé les contextes en quatre périodes. Pour plus de certitudes, nous nous sommes fondé uniquement sur les termini ante quos, c'est-à-dire sur la limite basse de la datation. Cette date représente en effet le moment à partir duquel nous sommes certains que la monnaie a été déposée (au sens archéologique). Cela n'exclut pas que la pièce ait été déposée plus tôt, notamment pour les contextes à datation imprécise ou pour les sites à occupation longue, où peuvent se poser des problèmes de résidualité. Mais cela resterait impossible à démontrer, et il nous semble préférable de nous en tenir uniquement aux données sûres.

- Période 1 : elle regroupe les monnaies déposées avant la guerre des Gaules, c'est-à-dire avant 60/50 av. J.-C. Elle représente les périodes La Tène D1 et D2a, qui vont de 150 à $60 / 50$ av. J.-C.

- Période 2 : elle regroupe les monnaies déposées entre la guerre des Gaules et le début de l'époque augustéenne. Elle correspond à La Tène D2b (60/50 à $30 / 20$ av. J.-C.).

- Période 3 : elle regroupe les monnaies déposées à la période augustéenne, entre 30/20 av. J.-C. et 15/20 apr. J.-C. ${ }^{12}$.

- Période 4 : elle regroupe toutes les monnaies déposées après la période augustéenne, jusqu'au début du IIe siècle apr. J.-C. que nous avons choisi comme limite de notre enquête.

Une carte a été réalisée pour chacune de ces périodes, afin de saisir d'éventuelles dynamiques régionales.

\section{Période 1 (150 à 60/50 av. J.-C.)}

Sur les deux monnaies qui peuvent être assignées à cette période, un seul contexte est sans aucun doute antérieur à la conquête césarienne : il s'agit de l'obole massaliète trouvée à Epiais-Rhus, dans une fosse d'un secteur d'habitat (cat. 1), un contexte de peu postérieur à l'émission si on retient la datation proposée $^{13}$. Il n'est pas exclu que le contexte de Bibracte qui a livré la monnaie au cheval galopant de la vallée du Rhône (cat. 2) soit contemporain de la guerre des Gaules, d'autant plus que la stratigraphie de ce secteur pose problème.

12. Sur le plan archéologique, la fin du règne d'Auguste et le début du règne de Tibère sont difficiles à distinguer.

13. Feugère, Py 2011, p. 44 : l'émission y est datée de 200/125 av. J.-C. 


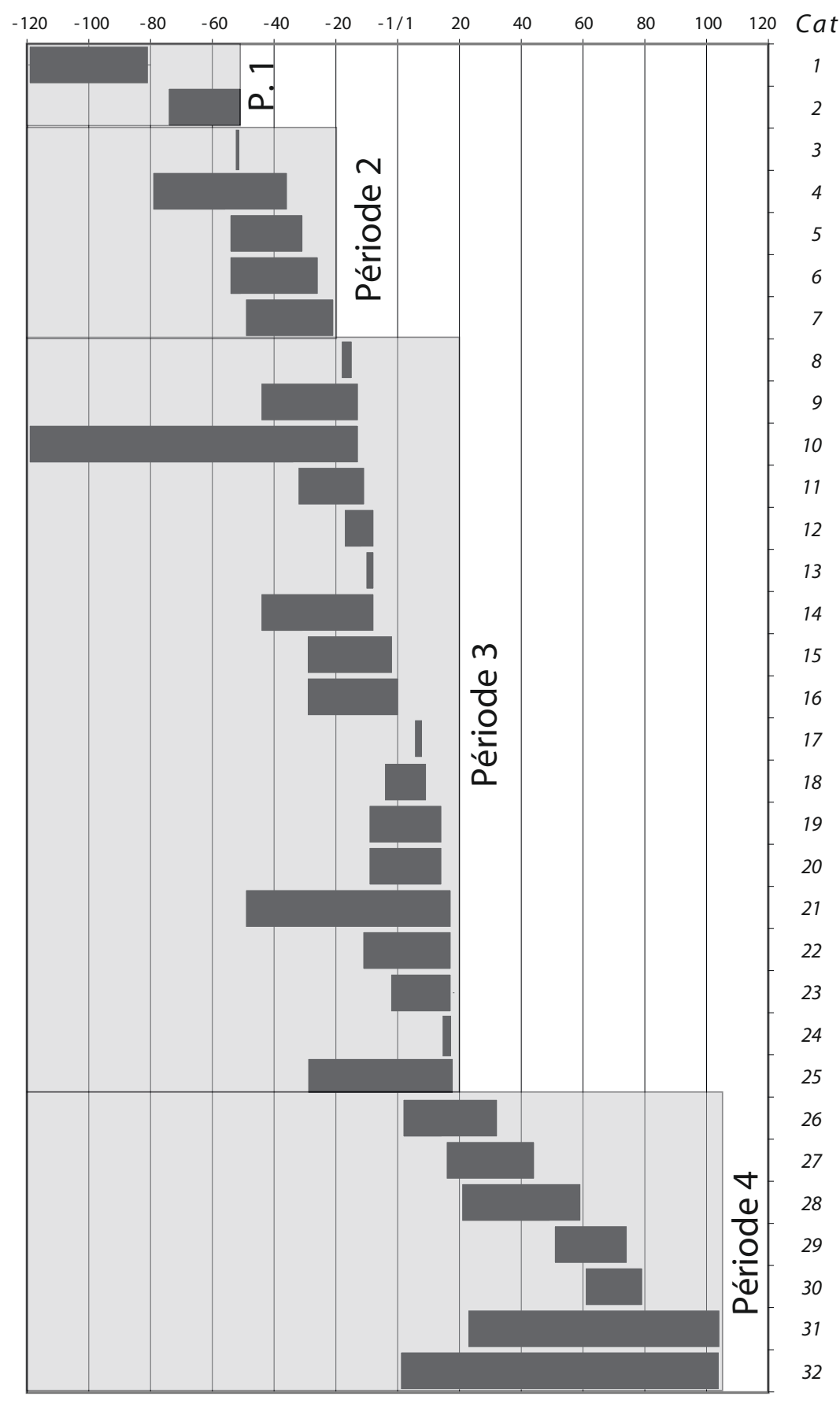

Figure 2 - Datations des contextes étudiés. 


\section{Période 2 (60/50 à 30/20 av. J.-C.)}

C'est véritablement à cette période que l'on commence à trouver en nombre important des monnaies de Méditerranée (39 pièces sur cinq sites différents). Le siège d'Alésia (cat. 3) en livre un assez grand nombre ${ }^{14}$, mais le contexte archéologique et historique très particulier interdit d'y voir une situation normale.

Ce sont surtout des oboles de Marseille que l'on retrouve, quelles que soient les régions (cat. 4 à 7), et parfois en grand nombre comme à La Chaussée-Tirancourt. Les monnaies qui ne viennent pas de Gaule méridionale sont italiques (trois drachmes padanes à Alésia) ou illyrienne (au Titelberg).

\section{Période 3 (30/20 av. J.-C. à 15/20 apr. J.-C.)}

Même si on ne retrouve plus de concentration importante comme à la période 2, l'époque augustéenne marque la diffusion la plus importante des monnaies méditerranéennes ( 21 monnaies réparties sur 15 sites). Les oboles massaliètes (6 ex.) sont maintenant concurrencées par les monnaies de la vallée du Rhône, essentiellement du type au cavalier (7 ex.). Deux monnaies viennent d'Italie centrale, et semblent appartenir aux séries dites «pseudo-Ebusus/Massalia» récemment identifiées par $\mathrm{C}$. Stannard et $\mathrm{S}$. Frey-Kupper. Le bronze de Trèves (cat. 18) appartiendrait au groupe $\mathrm{C}$, qui est attesté à Pompéi dans des niveaux antérieurs à 89 av. J.-C., tandis que celui de Sermuz (cat.9) appartiendrait au groupe $\mathrm{F}$, qui pourrait être daté du «deuxième quart du rer siècle av. J.-C.» ${ }^{15}$. L'exemplaire de Sermuz, le premier à avoir un semblant de contexte archéologique, ne s'y oppose pas ${ }^{16}$. Enfin, le bronze d'Antiochos III (cat. 23) trouvé en contexte augustéen montre que toutes les découvertes de monnaies des Ive et IIIe siècle av. J.-C. ne sont pas contemporaines de leur date d'émission.

Il n'est pas exclu que certaines pièces aient été déposées plus tôt (cat. 14, 21 et surtout 10).

14. Dont il faut probablement exclure le bronze à légende SEX F/T POMP, qui semble émis postérieurement.

15. FreY-KuPPER, StANNARD 2010,p. 121-122 et 131-133 ; STANNARD à paraître (la pièce cat. 9 correspond à la combinaison de types 34 , la pièce cat. 18 à la combinaison 3). Les identifications données ici se basent uniquement sur la bibliographie : S. Frey-Kupper et Cl. Stannard insistent sur l'importance de voir les pièces elles-mêmes pour donner une détermination définitive, ce qu'il n'a pas été possible de faire dans le cadre de cette étude.

16. Les monnaies du site proviennent de récoltes de surface, mais des sondages archéologiques ont livré du mobilier en place qui permet de cerner la durée d'occupation, courte, entre 50/40 et $15 / 10$ av. J.-C. 

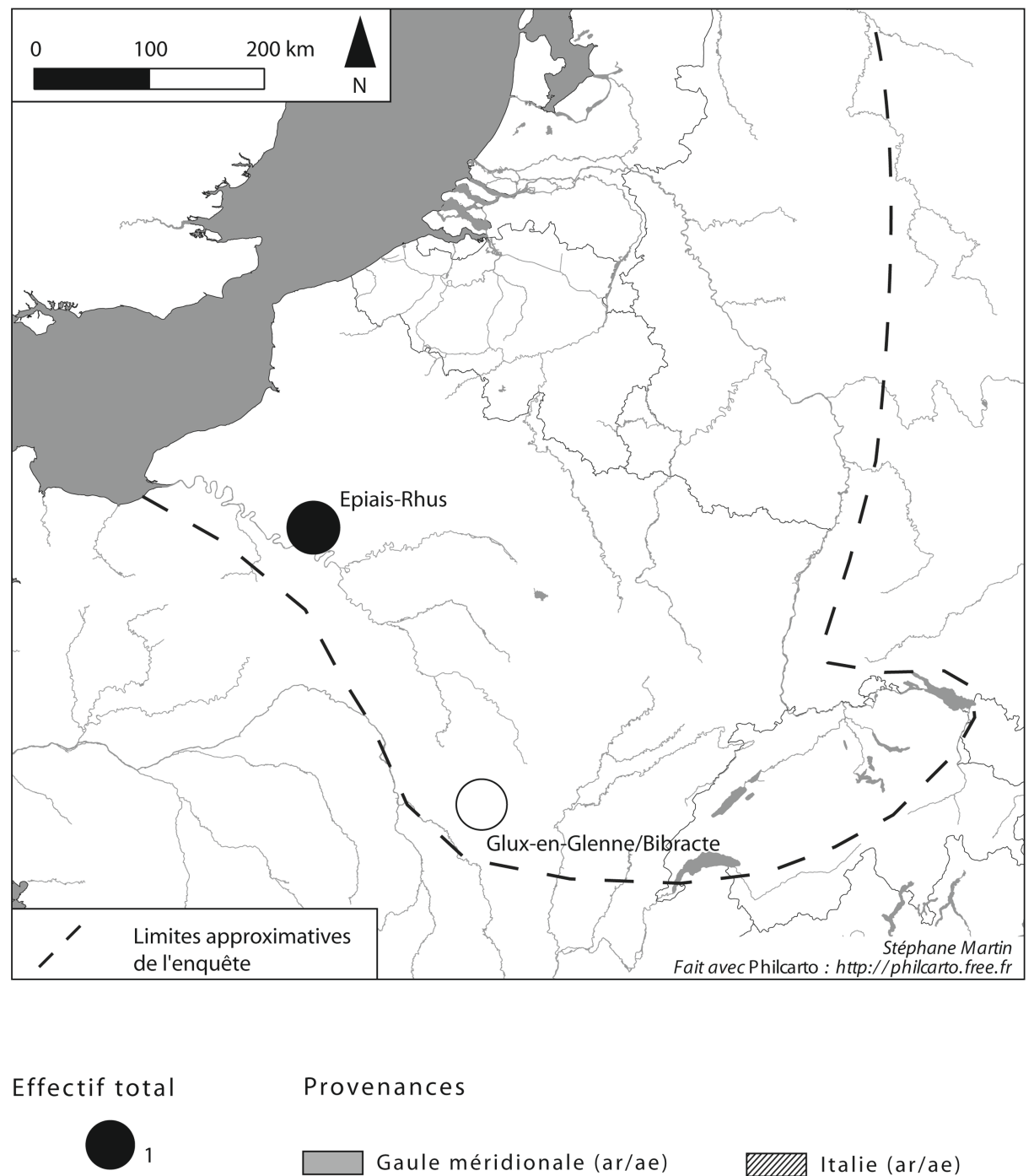

\section{Provenances}

\begin{tabular}{|c|c|c|}
\hline Gaule méridionale (ar/ae) & VIIII & Italie (ar/ae) \\
\hline Marseille (ar) & & Illyrie (ae) \\
\hline Marseille (ae) & 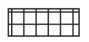 & Syrie (ae) \\
\hline Vallée du Rhône (ar) & $:::::$ & Numidie (ar) \\
\hline
\end{tabular}

Figure 3 - Carte des contextes de la période 1. 

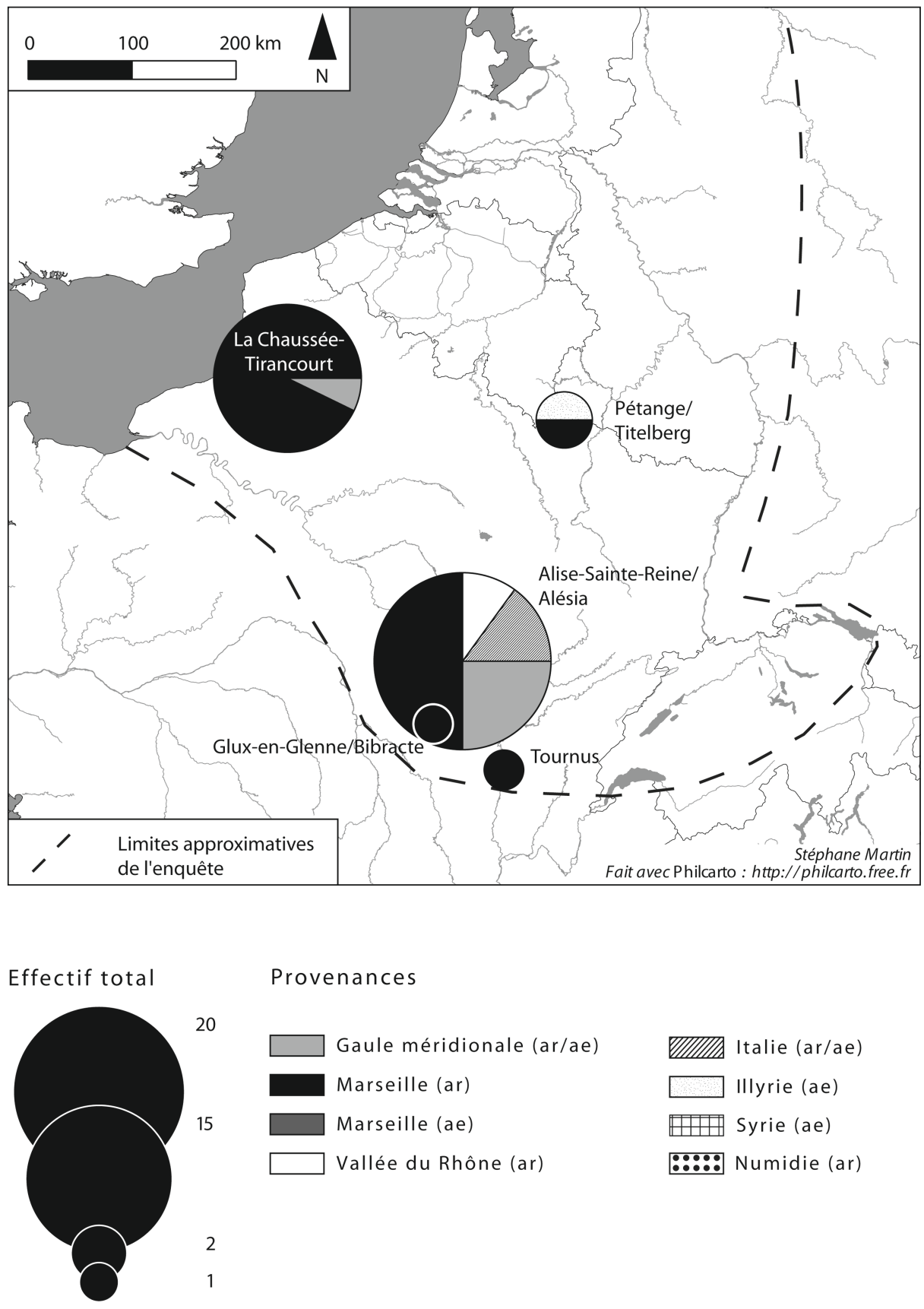

\section{Provenances}

\begin{tabular}{ll}
\hline Gaule méridionale (ar/ae) & \\
Marseille (ar) & $\square$ Italie (ar/ae) \\
\hline Marseille (ae) & \\
$\square$ Vallée du Rhône (ar) & \\
\hline
\end{tabular}

Figure 4 - Carte des contextes de la période 2. 

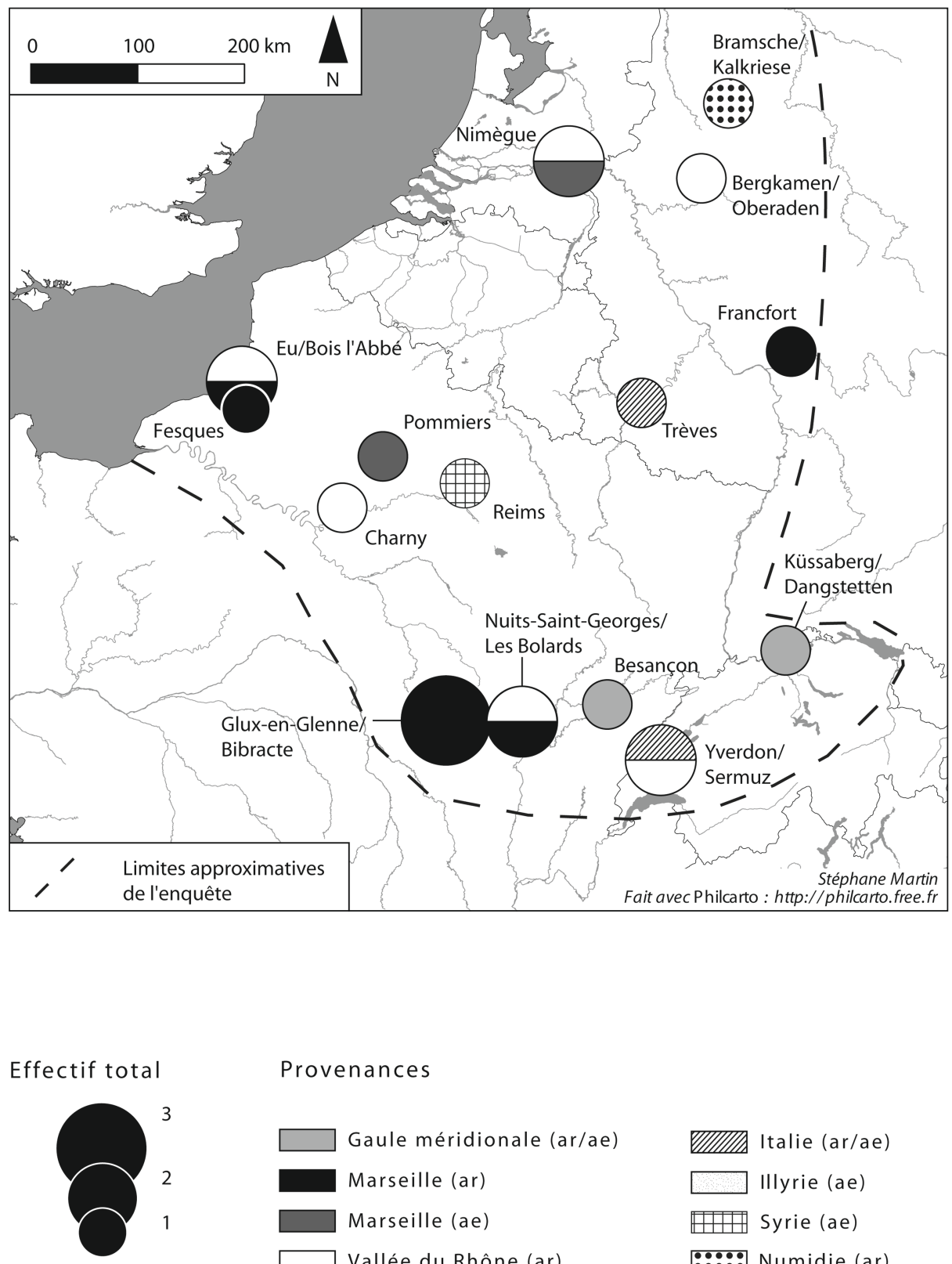

Provenances

\begin{tabular}{|c|c|c|}
\hline Gaule méridionale (ar/ae) & $\mathbb{Z}$ & Italie (ar/ae) \\
\hline Marseille (ar) & & Illyrie (ae) \\
\hline Marseille (ae) & 世埵 & Syrie (ae) \\
\hline Vallée du Rhône (ar) & $: \because:::$ & Numidie (ar) \\
\hline
\end{tabular}

Figure 5 - Carte des contextes de la période 3. 

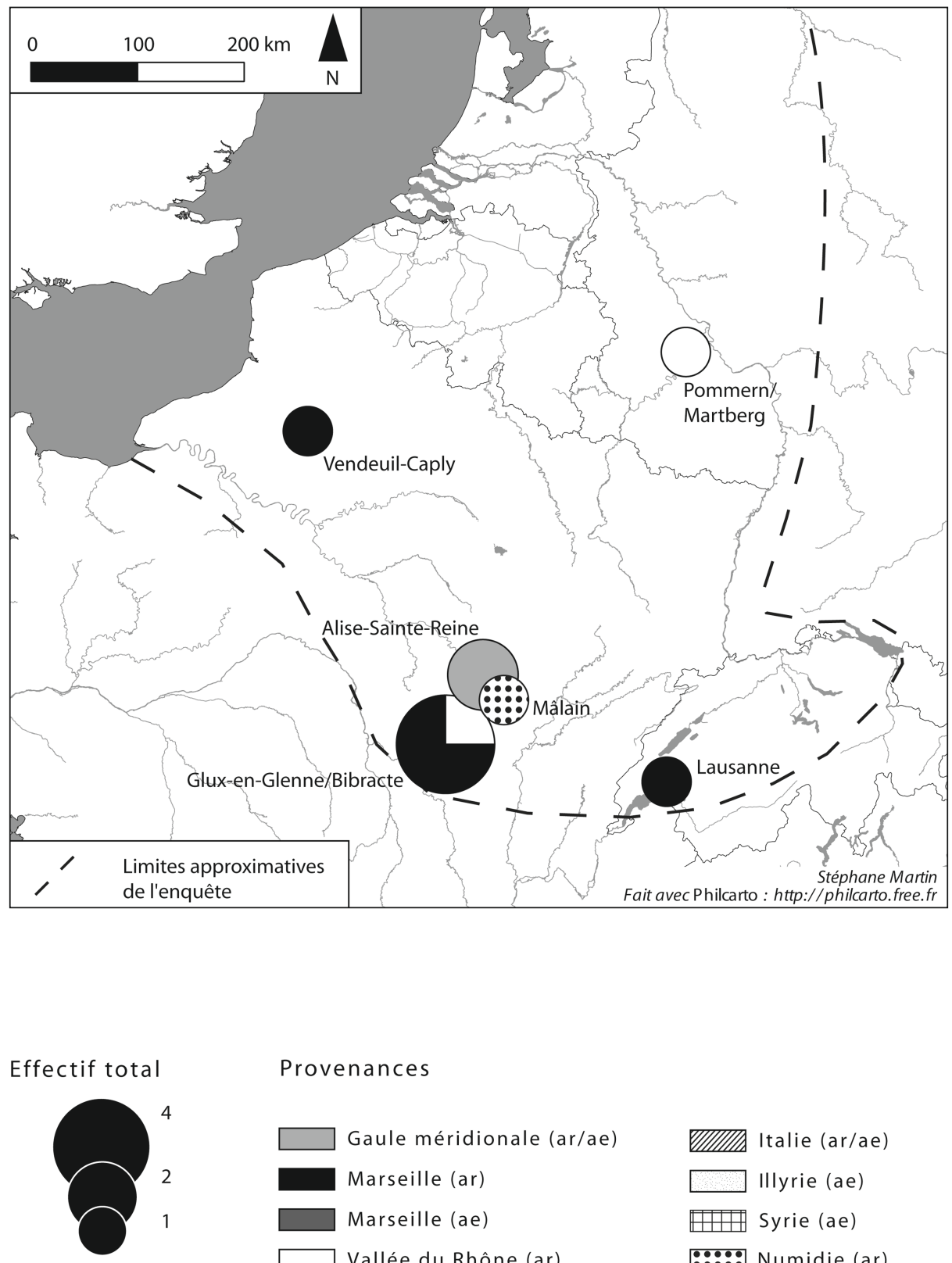

Provenances
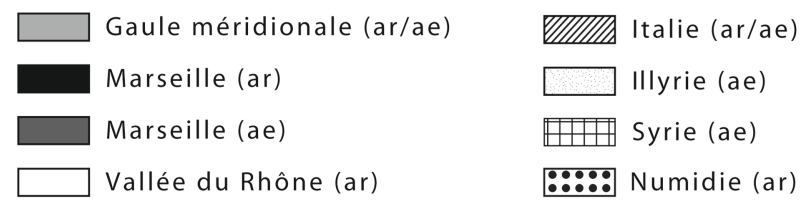

Figure 6 - Carte des contextes de la période 4. 


\section{Période 4 (15/20 à 100 apr. J.-C.)}

Après la période augustéenne, la diffusion des monnaies méridionales se contracte fortement (dix monnaies pour sept sites). Les types sont les mêmes qu'à la période précédente, et la plupart des exemplaires doivent selon nous être considérés comme résiduels. À Vendeuil-Caply comme au Martberg (cat. 29 et 30), on se trouve dans le cas d'une reconstruction du sanctuaire, sur une zone occupée dès l'époque laténienne. À Bibracte, l'occupation cesse à l'époque tibérienne, et une partie des monnaies de l'état final de la Fontaine Saint-Pierre provient probablement du brassage des états précédents (cat. 31).

Comment interpréter ces données? Plusieurs constatations s'imposent. Tout d'abord, la majorité des exemplaires répertoriés proviennent de Gaule méridionale, et il s'agit essentiellement de pièces en argent (oboles de Marseille, deniers de la vallée du Rhône pour la plupart), quelles que soient les périodes. Il est évident que, par leur valeur intrinsèque, ces monnaies s'inséraient mieux dans la circulation locale, d'autant plus que pour ce métal, les étalons pondéraux d'une grande partie de la Gaule septentrionale étaient équivalents ${ }^{17}$. L'influence du couloir rhodanien semble importante, et on note toujours, au débouché du Rhône et de la Saône, dans le Centre-Est et en Suisse, une petite concentration qui illustre l'importance de cet axe, que nous évoquions plus haut.

Les monnaies de bronze, comme les monnaies frappées hors de Gaule, n'arrivent qu'à partir de la guerre des Gaules. Le demi-siècle qui suit la conquête se caractérise par l'explosion du nombre de monnaies méditerranéennes dans les contextes, comparé aux deux seules monnaies de la période 1. Il nous semble difficile d'échapper à la conclusion que l'annexion des Gaules par Rome a précipité la circulation de ces monnayages exogènes. Les contextes de découverte, par ailleurs, semblent confirmer qu'il existe un lien fort entre la présence de pièces méditerranéennes et la présence de militaires, romains ou liés à l'armée romaine. En effet, à la période 2, outre Alésia, Bibracte, le Titelberg et La Chaussée-Tirancourt sont trois oppida connus pour avoir hébergé des troupes ${ }^{18}$. Avec une forte proportion d'oboles massaliètes, le dernier site présente par ailleurs un faciès général proche de deux autres sites peu éloignés, Liercourt-Erondelle et Pommiers, qui se trouvent être également des oppida ${ }^{19}$. Le site de Sermuz, rattaché ici à la période 3 , offre une

17. GRUEL 2005.

18. On sait que César a séjourné à Bibracte, et les fouilles ont mis au jour au Titelberg et à la Chaussée-Tirancourt les traces nettes d'une présence militaire romaine postérieure à la guerre des Gaules.

19. Delestrée, Boisard 2010 et Guichard et al. 1993,p. 54-55. Ces monnaies ne proviennent pas de contextes archéologiques, ce qui explique qu'elles ne soient pas prises en compte dans notre catalogue. Sur la Chaussée-Tirancourt, outre Delestrée 1997 (monnaies de fouille) voir également Delestrée, Boisard, Boulenger 2006 (monnaies de prospection, dont les conditions de récolte apparaissent opaques à la lecture de l'article). 
situation similaire. Ceci accrédite encore un peu plus l'idée selon laquelle les troupes romaines, après la conquête césarienne et avant le début des campagnes de Germanie, étaient stationnées sur les oppid ${ }^{20}$. Bon nombre de sites de la période 3 offrent également un lien direct avec l'armée : Nimègue, Oberaden, Francfort/ Höchst et Dangstetten sont des camps bien caractérisés.

Étant donné l'origine des monnaies frappées hors de Gaule (Italie centrale, plaine du Pô, Illyrie ${ }^{21}$ ), il est tentant de faire le lien, qu'ont déjà fait d'autres auteurs, avec la provenance des troupes romaines. D'autant plus que les monnaies padanes d'Alésia sont presque les seules connues en Gaule septentrionale ${ }^{22}$, et que les monnaies pseudo-Ebusus/Massalia d'Italie centrale semblent très peu diffusées hors de leur zone de frappe ${ }^{23}$. Une arrivée par coin drift est donc hautement improbable, et il faut plutôt supposer que ces pièces sont arrivées directement d'Italie dans des bourses de soldats.

À cela, il faut ajouter que le rythme d'arrivée des monnaies méditerranéennes dans la région étudiée semble suivre celui des monnaies romaines ${ }^{24}$. Les premières monnaies romaines, également en argent, arrivent au tout début du er siècle av. J.-C. (soit à La Tène D1b), à Gournay-sur-Aronde et Bibracte, et se font plus nombreuses à La Tène D2a, dans les décennies précédant l'arrivée de César. Mais cette dernière semble marquer une réelle rupture, avec la diffusion notamment de monnaies romaines en bronze, auparavant très rares. Il nous semble également que l'activité militaire en est la principale explication. Enfin, le tassement dans la diffusion des monnaies méditerranéennes après l'époque augustéenne correspond à la disparition progressive des pièces gauloises dans le stock monétaire, et l'affirmation des frappes impériales, avec entre autres la diffusion très importante de la deuxième série à l'autel de Lyon.

Que penser, dans ces conditions, de l'opinion de J.-M. Doyen citée au début de notre étude ? Est-il réellement possible d'identifier une circulation de «monnaies de bronze grecques (au sens large) [...] [dès] LT D2a, comme petit numéraire à part entière, en dehors de toute influence romaine » ? À n'en pas douter, des monnaies méditerranéennes, en bronze comme dans d'autres métaux, ont circulé en Gaule interne avant la guerre des Gaules : les importations sont déjà nombreuses à cette période, et il n'y a aucune raison que des monnaies n'y aient

20. Voir entre autres FichtL 1998.

21. Ces deux dernières régions faisaient partie du proconsulat attribué à César en 59 av. J.-C. par la lex Vatinia.

22. ArsLan (ed.) 2010, p. 29-33 : outre Alésia, on note un (ou plus) exemplaire dans la région de Metz, et des exemplaires plus nombreux en Suisse transalpine. Pour ces derniers, comme pour les exemplaires de Bavière, on peut estimer qu'il s'agit de la frange septentrionale de la distribution «normale» de ces monnaies. Les pièces de Gaule interne ou de Grande-Bretagne apparaissent comme des anomalies.

23. Frey-Kupper, Stannard 2010, p. 135-143 ; Feugère, Py 2011, p. 421.

24. Pour une esquisse plus complète, voir MarTin 2011. 
pas été associées, même si les découvertes en contexte sont encore très rares ${ }^{25}$. De même, les régions côtières (les façades atlantique et méditerranéenne) ont certainement été plus exposées à l'arrivée de monnaies étrangères, et de façon plus précoce $^{26}$ (il faut souligner néanmoins que la recherche s'est focalisée sur ces territoires). Cependant, en Gaule septentrionale, il est clair que la conquête a précipité l'arrivée de telles monnaies ${ }^{27}$. Pour autant, l'archéologie nous interdit d'y voir le résultat d'un désordre dans la circulation monétaire, qui aurait entraîné l'acceptation par les Gaulois de n'importe quel numéraire ${ }^{28}$. Au contraire, les contextes archéologiques montrent des concentrations de monnaies méditerranéennes sur des sites bien précis, et tout permet de penser qu'elles y ont été apportées par des usagers méditerranéens. Nous privilégions donc l'hypothèse militaire ${ }^{29}$, dont il faut reconnaître qu'elle a une visibilité archéologique et littéraire importante.

Bien qu'il reste selon nous un facteur d'explication marginal, le rôle possible des marchands ne doit toutefois pas être négligé. Le récit de César indique clairement que la Gaule était parcourue par des mercatores (des marchands professionnels) ${ }^{30}$, dont l'origine n'est jamais précisée. Il atteste également que des negotiatores $^{31}$, c'est-à-dire des hommes d'affaires, citoyens romains, étaient établis

25. Le dépôt de quatre monnaies grecques d'Esneux (B), dont les plus récentes sont du II siècle av. J.-C., pourrait en être un témoin. Le contexte de découverte est malheureusement très flou, même si la provenance ne fait pas de doute : BAR 1991,p. 131-134. Hors de la zone étudiée ici, dans le centre de la France, on note deux cas publiés récemment de monnaies méditerranéennes dans des contextes anciens : un bronze punique de Sardaigne sur un site occupé uniquement dans la première moitié du IIIe siècle av. J.-C. à Mer (TRÉBuchet et al. 2009) et un bronze sicilien de Hiéron II dans un contexte 130-110 av. J.-C. à Orléans (Gruel et al. 2009).

26. C'est particulièrement clair en Gaule du sud, pour laquelle on dispose de quelques contextes de découvertes, et d'un inventaire qui prend en compte l'arrière-pays. Les monnaies grecques, magno-grecques et africaines se concentrent assez clairement sur le littoral : FeUGÈre, Py 2011, en particulier p. 400, 405, 407, 417.

27. Bien que ne disposant pas de contextes archéologiques précis, M. Bar, en étudiant l'environnement archéologique général (en se basant notamment sur les dates connues de début d'occupation des sites), arrive à des conclusions similaires pour les monnaies hellénistiques de Méditerranée occidentale (hors Espagne) : BAR 1991, p. 178-182 et 187-192.

28. Ce que J.-B. Colbert de Beaulieu (1973, p. 323 sqq.) qualifiait de «circulation secondaire». Pour Br. Fischer, c'était encore une des raisons majeures pour la circulation des monnaies africaines en Gaule : FISCHER 1978, p. 135-152. Mais le cadre interprétatif utilisé est maintenant largement abandonné, et son étude mériterait d'être reprise en intégrant les nouvelles données.

29. Auquel cas certaines monnaies méditerranéennes, associées à d'autres indices, pourraient constituer des traceurs de la provenance des troupes : KeMMERs 2009 propose cette grille d'analyse pour les monnaies gauloises dans les camps augustéens.

30. CÉSAR, $B G, \mathrm{I}, 1$ est très clair à ce sujet.

31. Le terme de negotiator n'apparaît jamais dans le texte ; César utilise toujours l'expression negotiandi causa. La différence entre mercator et negotiator est étudiée dans FEUVRIER-PRÉVOTAT 1981, à partir de l'œuvre de Cicéron. 
en Gaule interne ${ }^{32}$. Dans l'œuvre de Cicéron, de tels personnages sont toujours proches des milieux dirigeants romains, et César mentionne un chevalier romain dans les victimes du massacre d'Orléans. On peut donc restituer des emporia, occupés au moins partiellement par des Romains, aux endroits stratégiques ${ }^{33}$; les trois villes citées par César se trouvent d'ailleurs sur la Loire ou la Saône ${ }^{34}$. On notera que parmi les sites de notre catalogue où aucune influence militaire n'est décelée, plusieurs se situent sur des axes de communications fluviaux ou routiers ${ }^{35}$. Outre la diffusion des produits méditerranéens, principalement des amphores vinaires italiques dont l'importation connaît une véritable explosion à partir du dernier quart du IIe siècle av. J.-C. ${ }^{36}$, l'archéologie a peut-être identifié deux comptoirs de marchands, à Lyon et à Arnac-la-Poste ; ces deux gisements, établis tous les deux dans la deuxième moitié du IIe siècle av. J.-C., se caractérisent en effet par l'usage très précoce de techniques constructives méditerranéennes ${ }^{37}$.

Si des citoyens romains prenaient la peine de venir s'installer pour affaires en Gaule interne, on peut se demander à quel point les commerçants méditerranéens étaient à l'écart d'une route commerciale comme celle de l'Atlantique, même s'ils n'en avaient pas le contrôle. Selon toute vraisemblance, ces étrangers stationnés dans des comptoirs manipulaient des monnaies, et on peut supposer qu'ils retournaient périodiquement dans leur patrie d'origine. Mais en rapportaient-ils des monnaies, ou bien les changeaient-ils contre du numéraire local ?

Ce point nous amène, pour conclure, à dire un mot sur la fonction des monnaies méditerranéennes que nous trouvons en Gaule septentrionale. Pour J.-M. Doyen, les pièces en bronze pouvaient circuler «comme petit numéraire à part entière.» A priori, les contextes archéologiques des exemplaires que nous avons répertoriés, pour le bronze comme pour l'argent, ne laissent pas entrevoir de pratiques de

32. Comme le précise César pour les negotiatores de Cenabum/Orléans (BG, VII, 3) et Cabillonum/ Châlons-sur-Saône (ibidem, VII, 42) ; on peut supposer que c'est également le cas pour ceux de Noviodunum (ibid., VII, 55 : peut-être Nevers). Cl. Feuvrier-Prévotat (1981) a montré que c'est également le cas pour les negotiatores mentionnés par Cicéron.

33. Le célèbre passage du Pro Fonteio de Cicéron (V,11) concerne la Gaule transalpine. Au-delà de la probable exagération due au contexte judiciaire, il permet néanmoins de montrer combien l'implication des hommes d'affaires romains pouvait être importante et rapide dans une province nouvellement conquise. Tout aussi intéressant, mais moins cité, un passage de Salluste nous donne à voir les contacts entre un commerçant romain et les élites gauloises : Catilina, XL, 2 : « [P.] Umbrenus, qui avait fait du négoce en Gaule, était connu de la plupart des chefs de cités et les connaissait lui-même.» Il s'agit au moins de la Gaule transalpine (puisque l'affranchi dont il est question est chargé de rencontrer les émissaires allobroges à Rome), peut-être de la Gaule chevelue.

34. Les sources anciennes nous apprennent d'ailleurs que les Eduens percevaient des portoria sur cette rivière : FRANCE 2001,p. 216-221.

35. Epiais-Rhus est proche de la confluence entre l'Oise et la Seine ; Tournus est sur le cours de la Saône ; Les Bolards sont situés sur la voie qui mène de Châlons-sur-Saône à Langres, en passant par Dijon.

36. Poux 2004, notamment p. 192-212.

37. Poux, Savay-Guerraz (dir.) 2003, p. 124-145 ; Toledo i Mur 1997-1998. 
déposition particulières, liées à la provenance des pièces. Mais il est probable, comme nous venons de le noter, qu'elles ont été manipulées principalement par des Méditerranéens. Quelle a pu être l'attitude des usagers locaux devant ces monnaies ? On rappellera ici un victoriat romain retrouvé sur l'oppidum de La Cloche près de Marseille, qui était clairement en train d'être retravaillé, probablement en vue d'une refonte ${ }^{38}$. Si les monnaies en argent, même d'un étalon pondéral proche de l'étalon local, pouvaient servir de source de métal, comment interpréter les monnaies que nous retrouvons, et plus particulièrement celles en bronze ? Monnaies en circulation? Souvenirs ? Jetons ? Les exemplaires retrouvés sont-ils la norme, ou la plupart étaient-ils refondus ${ }^{39}$ ? Bien souvent, ni le contexte archéologique, ni les analyses ne permettent une interprétation définitive, et les textes ne permettent pas de connaître les espèces employées lors des échanges. Reconnaissons à ce sujet notre impuissance ${ }^{40}$.

\section{CATALOGUE}

Les contextes sont classés par ordre chronologique, et regroupés selon les quatre grandes périodes utilisées dans le texte. Pour les sites français, on a indiqué lorsqu'elle était disponible la notice concernant le contexte de découverte de la Carte archéologique de la Gaule (abrégée $C A G$ et suivie du numéro de département). Les monnaies de Gaule méditerranéenne sont classées selon FeugÈre, Py 2011, avec l'équivalence à l'Atlas de La Tour pour la plupart des exemplaires ; les monnaies de la Vallée du Rhône sont classées d'après Deroc 1983. Pour les autres exemplaires, on s'est contenté d'une ou deux références usuelles. La description précise des monnaies cataloguées est généralement accessible dans la bibliographie citée.

\section{Période 1 (déposition antérieure à 60/50 av. J.-C.)}

1. Epiais-Rhus, Val-d'Oise, F ; habitat, phase LT D1b (120-80 av. J.-C.) : 1 obole de Marseille (OBM-9c).

Bibliographie : CAG 95, p. 229-233 ; LARDY et al. 1987, p. 154.

38. Снавот 1985, p. 52-53, monn. 7.

39. En Croatie, cette dernière hypothèse est parfois privilégiée les monnaies nord-africaines qu'on y retrouve en quantité, principalement dans des dépôts de type «Mazin» (du nom de la trouvaille la plus importante) : DuBOLNIĆ GLAVAN, GlavAš 2011 (avec bibliographie antérieure). Pour la Belgique moderne : BAR 1991, passim (en particulier p. 235-241). Plus largement, pour des exemples de manipulations diverses à partir d'objets méditerranéens dans le monde celtique, voir VERGER 2011 sur les vases en bronze.

40. Je tiens à adresser mes vifs remerciements, dans l'ordre alphabétique, à M. Carrive, J.-M. Doyen, S. Frey-Kupper, J. Genechesi, E. Hiriart, St. Izri, P. Nouvel, M. Reddé, Cl. Stannard, A. Tonc, ainsi qu' au comité de lecture de la Revue Numismatique, pour les remarques, corrections, données et manuscrits inédits ou à paraitre qu'ils ont bien voulu me faire parvenir et qui ont contribué à enrichir ce travail. Toutes les erreurs et insuffisances restent de mon fait. 
2. Glux-en-Glenne, Nièvre, $\mathbf{F}$; oppidum de Bibracte, Porte du Rebout, phase 4 Nord (75-50 av. J.-C.) : 1 monnaie au cheval galopant de la Vallée du Rhône (DEROC 1983, «cheval galopant», groupe II).

Bibliographie : Buschenschutz et al. (dir.) 1999, p. 198, monn. 59 ; Gruel, Popovitch 2007, non répertorié.

\section{Période 2 (déposition antérieure à 30/20 av. J.-C.)}

3. Alise-Sainte-Reine, Côte-d'Or, F ; siège d'Alésia (52 av. J.-C.) : 2 drachmes à la croix (peut-être DCR-112 et DCR-119); 1 denier attribué aux Rutènes (LT $3433=$ RUT-206A) ; 1 bronze à légende NAMA $\Sigma \Sigma$ AT (LT $2698=$ NIM-2698) ; 1 bronze à légende SEX F/T POMP (LT $4353=$ TPS-4353); 10 oboles de Marseille (1 OBM-7, 2 OBM-10, 7 OBM-8/10) ; 2 deniers au cavalier de la Vallée du Rhône (DEROC 1983, «cavalier», groupe indéterminé) ; 3 drachmes padanes à légende oıxol (ARSLAN 1990, type 15).

Bibliographie : $C A G$ 21/1,p. 269-291 ; Reddé, SCHNURBEIN (dir.) 2001, vol. 2, p. 11-39, monnaies 617-629 et 665-671; ARSLAN (ed.) 2010, p. 29.

4. Glux-en-Glenne, Nièvre, $\mathbf{F}$; oppidum de Bibracte, Pâture du Couvent 1 , secteur B, phase 2 (80-40/30 av. J.-C.) : 1 obole de Marseille (OBM-8).

Bibliographie : FleIscher 2007, vol.2, p. 41 ; Gruel, PoPOvitch 2007,p. 153, monn. 4.

5. Pétange, canton d'Esch-sur-Alzette, LUX ; oppidum du Titelberg, secteur central, phase LT D2b (60/50-30 av. J.-C.) : 1 bronze de Dyrrachium (BMC 7, 162 ; SNG United Kingdom, vol. VII, 848) ; 1 obole de Marseille (OBM-8/11). Bibliographie : MetzLER 1995, p. 164 et 633.

6. Tournus, Saône-et-Loire, F ; lieu-dit Clos-Roy, phase LT D2b (60/5030/20 av. J.-C.) : 1 obole de Marseille (OBM-8/11).

Bibliographie : $C A G$ 71/4,p. 462-463 ; VAUSSANVIN 1987, p. 243, monn. 2.2.14.

7. La Chaussée-Tirancourt, Somme, $\mathbf{F}$; porte principale de l'oppidum, phases I-II (50-20 av. J.-C.) : 1 bronze à légende VOLCA/AREC (LT $2677=$ VLC2677) ; 14 oboles de Marseille (dont 3 OBM-8/11 : les références typologiques précises des oboles ne sont pas précisées ; seules trois pièces sont illustrées). Bibliographie : DeLESTRÉE 1997.

\section{Période 3 (déposition antérieure à 10/20 apr. J.-C.)}

8. Nimègue, province de Gueldre, NL ; camp augustéen de la Hunerberg (19-15/12 av. J.-C.) : 1 denier au cavalier de la Vallée du Rhône (DEROC 1983, «cavalier», groupe IV/23); 1 petit bronze de Marseille (LT 1673).

Bibliographie : KEMMERs 2006, p. 279. 
9. Yverdon-les-Bains, canton de Vaud, CH ; oppidum de Sermuz (50/4015/10 av. J.-C.) : 1 denier au cavalier de la Vallée du Rhône (Deroc 1983, «cavalier», groupe IV/23) ; 1 petit bronze pseudo-Ebusus/Massalia (LT 1969). Bibliographie : BrunetTy, Curdy 2007, p. 589, monn. 1 et 47.

10. Nuits-Saint-Georges, Côte-d'Or, F ; sanctuaire des Bolards, période I, état 1 (120-15/10 av. J.-C.) : 1 obole de Marseille (OBM-8/11).

Bibliographie : $C A G$ 21/3, p. 5-22 ; POMMEREt (éd.) 2001, p. 155, monn. 1.

11. Eu, Seine-Maritime, F ; sanctuaire du Bois-l'Abbé, phase 1a, horizon 1 (35/30-10 av. J.-C.) : 1 denier au cavalier de la Vallée du Rhône (Deroc 1983, «cavalier», groupe IV/25).

Bibliographie : $C A G$ 76, p. 245-250 ; MANGARD 2008, p. 218, monn. 207.

12. Küssaberg, Bade-Wurttemberg, D ; camp légionnaire de Dangstetten (20/15-7 av. J.-C.) : 1 bronze ibéro-languedocien (LT 2403 var. = IBL-2403). Bibliographie : FInGERLIN 1986, p. 153, Fundstelle 424, obj. 2.

13. Bergkamen, Rhénanie-du-Nord - Westphalie, D ; camp légionnaire d'Oberaden (11-8/7 av. J.-C.) : 1 denier au cavalier de la Vallée du Rhône (Deroc 1983, «cavalier», groupe IV/26).

Bibliographie : KülHBORN 1992, p. 199, monn. 355.

14. Glux-en-Glenne, Nièvre, F ; oppidum de Bibracte, Fontaine-Saint-Pierre, état 2 (50/40-10/5 av. J.-C.) : 2 oboles de Marseille (OBM-8/11).

Bibliographie : Barral, Richard (dir.) 2009, p. 174 ; Gruel, Popovitch 2007, p. 153 , monn. 6 et 12 .

15. Pommiers, Aisne, F ; oppidum, niveau II (30-1 av. J.-C.) : 1 petit bronze de Marseille (LT 1673).

Bibliographie : $C A G$ 02, p. 351-354 ; Brun, DeBord 1991, p. 45, monn. 1 ; PION 1996, vol. 4, fig. 79.

16. Besançon, Doubs, F ; Parking de la Mairie, phase 3 (30-1 av. J.-C.) : 1 bronze à légende SEX F/T POMP (LT 4353 = TPS-4353).

Bibliographie : $C A G$ 25, p. 216-217 ; Guilhot, Goy (dir.) 1992, p. 125 , monn. 83.

17. Bramsche, Basse-Saxe, D ; lieu-dit Kalkriese, lieu probable de la clades Variana (9 apr. J.-C.) : 1 denier de Juba I ${ }^{\text {er }}$ (MaZard 1955, no 84, AlexanDROPOULOS 2000, no 29).

Bibliographie : BERGER 1996, p. 100, monn. C159. 
18. Trèves, Rhénanie-Palatinat, D ; ville romaine, horizon 1 (5 av.-10 apr. J.-C.) : 1 petit bronze pseudo-Ebusus/Massalia (LT 2227 var.).

Bibliographie : MoRSCHEISER-NIEBERGaLL 2009, p. 254, monn. 388.

19. Charny, Seine-et-Marne, $\mathbf{F}$; lieu-dit Les Champs de Choisy, enclos précoces (10 av.-15 apr. J.-C.) : 1 denier au cavalier de la Vallée du Rhône (DEROC 1983, «cavalier», groupe IV/23).

Bibliographie : $C A G$ 77/1, p. 359-362 ; SÉGUIER, MALLET 2005, p. 544.

20. Eu, Seine-Maritime, F ; sanctuaire du Bois-l'Abbé, phase 1a, horizon 2 (10 av.-15 apr. J.-C.) : 1 obole de Marseille (réf. indét.).

Bibliographie : $C A G$ 76, p. 245-250 ; MANGARD 2008, p. 219, monn. 241.

21. Fesques, Seine-Maritime, F ; sanctuaire du Mont du Val aux Moines, scellement de l'état IV (50 av.-15/20 apr. J.-C.) : 1 obole de Marseille (OBM-8/11). Bibliographie : $C A G$ 76, p. 269-273 ; MANTEL (éd.) 1998, p. 287, monn. 6.

22. Francfort-sur-le-Main, Hesse, D ; quartier de Höchst, camp militaire (12 av.15/20 apr. J.-C.) : 1 obole de Marseille (OBM-2c).

Bibliographie: KUвON 2011, p. 140, monn. 5.

23. Reims, Marne, $\mathbf{F}$; fouilles du tramway, état 1 (5/1 av.-15/20 apr. J.-C.) : 1 bronze d'Antiochos III de Syrie (probablement SNG Israël 593-599).

Bibliographie : DoYen inédit, monn. 1.

24. Nuits-Saint-Georges, Côte-d'Or, F ; sanctuaire des Bolards, période II, construction (vers 15/20 apr. J.-C.) : 1 denier au cavalier de la Vallée du Rhône (DEROC 1983, «cavalier», groupe IV/26).

Bibliographie : $C A G$ 21/3, p. 5-22 ; POMMERET (éd.) 2001, p. 155, monn. 3.

25. Glux-en-Glenne, Nièvre, F ; oppidum de Bibracte, Pâture du Couvent 1 , secteur B, phase 3 (30 av.-vers 20 apr. J.-C.) : 1 imitation d'obole de Marseille ? (imitation d'OBA-M ? Pour K. Gruel, cette monnaie s'apparente à des types auvergnats).

Bibliographie : FleISCHER 2007, vol. 2, p. 54 ; GRUEL, PoPOVITCH 2007,p. 154, monn. 25.

\section{Période 4 (déposition postérieure à 15/20 apr. J.-C.)}

26. Alise-Sainte-Reine, Côte-d'Or, F ; zone du théâtre, phase 1 (jusqu'en 30/35 apr. J.-C.) : 1 bronze à légende VOLCA/AREC (LT $2677=$ VLC-2677). Bibliographie : $C A G$ 21/1,p. 382-388 ; Rossi (dir.) 2005, p. 88, monn. 1542-1. 
27. Lausanne, canton de Vaud, $\mathbf{C H}$; fouille de Chavannes 11, secteur A, horizon 5 (10/20-40/50 apr. J.-C.) : 1 imitation d'obole de Marseille en bronze (OBP-7).

Bibliographie : LuginbÜHL, SCHNEITER 1999, p. 311, monn. 1221.

28. Alise-Sainte-Reine, Côte-d'Or, F ; chantier XXX-XXXI (20-60 apr. J.-C.) : 1 bronze à légende SEX F/T POMP (LT $4353=$ TPS-4353).

Bibliographie : REDDÉ, SCHNURBEIN (dir.) 2001, vol. 1, p. 156 et vol. 2, p. 37 , monn. 672 .

29. Pommern, Rhénanie-Palatinat, $\mathbf{D}$; sanctuaire du Martberg, phase 7 de l'enclos (50-70/80 apr. J.-C.) : 1 denier au cavalier de la Vallée du Rhône (DEROC 1983, «cavalier», groupe indéterminé).

Bibliographie : NicKel et al. 2008, vol. 2, p. 624, monnaie 03/01/M084.

30. Vendeuil-Caply, Oise, F ; sanctuaire, construction du fanum (60-80 apr. J.-C.) : 1 obole de Marseille (OBM-8/11).

Bibliographie : CAG 60, p. 475-476 ; DelestréE 1985, p. 51.

31. Glux-en-Glenne, Nièvre, F ; oppidum de Bibracte, Fontaine-SaintPierre, état 3 (22/31-100/110 apr. J.-C.) : 1 denier au cavalier de la Vallée du Rhône (DEROC 1983, «cavalier», groupe III/20) ; 3 oboles de Marseille (OBM-8/11).

Bibliographie : BarRal, Richard (dir.) 2009, p. 171-172 ; GRUel, PoPovitch 2007, p. 153 , monnaies $8,10,11$ et p. 155 , monn. 37 .

32. Mâlain, Côte-d'Or, F ; remblais de destruction d'un mur augustéen (er siècle apr. J.-C.) : 1 denier de Juba I ${ }^{\text {er }}$ (MAZARD 1955, no 84, ALEXANDroPOULOS 2000, no 29).

Bibliographie : $C A G$ 21/2, p. 457-495 ; FISCHER 1978, p. 122, monn. 66.

\section{Bibliographie}

\section{Sources antiques}

CÉsAR, BG : César, Guerre des Gaules (éd. L.A. Constans revue et corrigée par A. Balland), Paris, Collection des Universités de France, série latine 32-33, 1995 (14éd.).

Cicéron, Pro Fonteio : Cicéron, Discours, tome VII (Pour M. Fonteius, Pour A. Cécina, Sur les pouvoirs de Pompée) (éd. A. Boulanger), Paris, Collection des Universités de France, série latine 54, 1961 ( $3^{\mathrm{e}}$ éd.).

Salluste, Catilina: Salluste, La Conjuration de Catilina. La Guerre de Jugurtha. Fragments des Histoires (éd. A. Ernout), Paris, Collection des Universités de France, série latine 98, 1941. 
AdAm 2007 : A.-M. AdAm, Les importations méditerranéennes en Gaule interne aux IVe et III siècle av. J.-C., dans La Gaule dans son contexte européen aux IVe et III siècle avant notre ère. Actes du XXVII colloque international de l'Association française pour l'étude de l'âge du fer, Clermont-Ferrand, 29 mai-

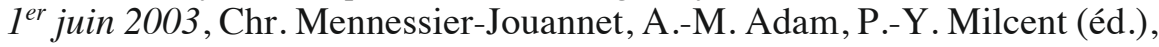
Lattes, 2007, p. 255-263.

Alexandropoulos 2000 : J. Alexandropoulos, Les monnaies de l'Afrique antique (400 av. J.-C.-40 apr. J.-C.), Toulouse, 2000.

Arslan 1990 : E. Arslan, Le monnayage celtique de la plaine du Pô (IveIer siècles av. J.-C.), Études Celtiques, XXVII, 1990, p. 71-102.

Arslan (ed.) 2010 : E. Arslan (ed.), Saggio di repertorio dei ritrovamenti di moneta celtica padana in Italia e in Europa e di moneta celtica non padana in Italia (version mise à jour le 11/12/2010), consulté le 12/11/2011 sur http://www .ermannoarslan.eu.

B AR 1991 : M. BAR, Monnaies grecques et assimilées trouvées en Belgique, (Travaux du Cercle d'études numismatiques 11), Bruxelles, 1991.

B AR 1996 : M. BAR, Monnaies grecques et assimilées trouvées en Belgique. Supplément I, Bulletin du Cercle d'études numismatiques, 33, 1996, p. 1-20.

B AR 2002 : M. BAR, Monnaies grecques et assimilées trouvées en Belgique. Suppl. II, Bulletin du Cercle d'études numismatiques, 39, 2002, p. 233-240.

Barral, Richard 2009 : Ph. Barral, H. Richard (dir.), Fouilles de la fontaine Saint-Pierre au Mont Beuvray (1988-1992, 1996) : aménagements d'une source sur l'oppidum de Bibracte, (Bibracte 17), Glux-en-Glenne, 2009.

BERGER 1996 : Fr. BERGER, Kalkriese 1. Die römischen Fundmünzen, (Römischgermanische Forschungen 55), Mayence, 1996.

Beyneix, Piot 1995 : A. Beyneix, C. Piot, Mobiliers grecs et de tradition grecques dans la vallée de la Garonne pendant les Âges du Fer, Aquitania, 13, 1995 , p. 33-73.

Brun, Debord 1991 : P. Brun, J. Debord, Monnaies trouvées trouvées en fouilles sur l'oppidum de Pommiers (Aisne), $R N, 1991$, p. 43-49.

Brunetti, Curdy 2007 : C. Brunetti, Ph. Curdy, Yverdon-les-Bains et Sermuz à la fin de l'âge du fer, (Cahiers d'Archéologie Romande 107), Lausanne, 2007.

Buschenschutz et al. 1999 : O. Buschenschutz, J.-P. GuIllaumet, I. RAlston (dir.), Les remparts de Bibracte : recherches récentes sur la porte du Rebout et le tracé des fortifications, (Bibracte 3), Glux-en-Glenne, 1999.

$C A G$ : Auteurs variés, Carte archéologique de la Gaule.Pré-inventaire archéologique publié sous la responsabilité de Michel Provost, Paris, Académie des Inscriptions et Belles-Lettre/Ministère de la Recherche/Ministère de la Culture, 1988-en cours.

Cнавот 1985 : L. Снавот, Monnaies étrangères aux émissions massaliètes découvertes sur l'oppidum de La Cloche, aux Pennes-Mirabeau (B.-du-Rh.), Documents d'archéologie méridionale, 8, 1985, p. 49-65.

Colbert de Beaulieu 1973 : J.-B. Colbert de Beaulieu, Traité de numismatique celtique I. Méthodologie des ensembles, (Annales littéraires de l'Université de Besançon 135), Paris, 1973. 
Delestrée 1985 : L.-P. Delestrée, Les monnaies gauloises du temple des «Châtelets» à Vendeuil-Caply (Oise), Revue archéologique de Picardie, 1/1, 1985, p. 51-64.

DelestréE 1997 : L.-P. Delestrée, Le numéraire gaulois témoin d'une présence militaire sur le site fortifié de la Chaussée-Tirancourt, Cah.Num., 131, mars 1997, p. 5-13.

Delestrée, Boisard 2010 : L.-P. Delestrée, Cl. Boisard, Les monnaies gauloises du camp militaire de Liercourt-Érondelle (Somme), dans L'armée et la monnaie II. Actes de la journée d'études du 25 avril 2009 à la Monnaie de Paris, D. Hollard (éd.), (Recherches et Travaux de la Société d'Études Numismatiques et Archéologiques 3), Paris, 2010, p. 21-42.

Delestrée, Boisard, Boulenger 2006 : L.-P. Delestrée, Cl. Boisard, D. Boulenger, Les monnaies gauloises du site fortifié de La Chaussée-Tirancourt (Somme) : coexistence d'un faciès militaire et d'un faciès indigène, dans L'armée et la monnaie. Actes de la journée d'études du 10 décembre 2005 à la Monnaie de Paris, D. Hollard (éd.), (Recherches et Travaux de la Société d'Études Numismatiques et Archéologiques 1), Paris, 2006, p. 7-25.

Deroc 1983 : A. Deroc, Les monnaies gauloises d'argent de la Vallée du Rhône, (Annales littéraires de l’Université de Besançon 281), Paris, 1983.

Doyen 2011 : J.-M. Doyen, Les monnaies d'Ebusus en Gaule du nord et en Bretagne : un faux «traceur» des campagnes césariennes ?, $R N, 2011$, p. 265-283.

DOYEN INÉDIT : J.-M. DoYEN, Monnaies grecques autonomes et romaines provinciales provenant des fouilles récentes de Reims (Marne), manuscrit inédit (état 2009).

Dubolnić Glavan, Glavaš 2011 : M. Dubolnić Glavan, V. Glavaš, Prilog poznavanju najstarijeg opticaja novca na prostoru primorske padine južnog Velebita (Contribution to the study of the oldest coin circulation on territory of Southern sub-Velebit littoral), Prilozi Instituta za arheologiju u Zagrebu, 28, 2011, p. 95-121.

Feugère, Py 2011 : M. Feugère, M. Py, Dictionnaire des monnaies découvertes en Gaule méditerranéenne : (530-27 av. notre ère), Montagnac, 2011.

Feuvrier-Prévotat 1981 : Cl. Feuvrier-Prévotat, Negotiator et mercator dans le discours cicéronien : essai de définition, Dialogues d'Histoire Ancienne, 7, 1981, p. 367-405.

FICHTL 1998 : St. FichTL, La présence militaire romaine sur les oppida dans la Gaule du nord et de l'est, dans Studien zur Archäologie der Kelten, Römer und Germanen in Mittel- und Westeuropa. Alfred Haffner, zum 60. Geburtstag gewidmet, A. Müller-Karpe et al. (éd.), (Internationale Archäologie. Studia honoraria 4), Rahden, 1998, p. 153-168.

FingerLIN 1986 : G. Fingerlin, Dangstetten. I, Katalog der Funde: Fundstellen 1 bis 603, (Forschungen und Berichte zur Vor- und Frühgeschichte in BadenWürttemberg 22), Stuttgart, 1986. 
FISCHER 1978 : Br. FISCHER, Les monnaies antiques d'Afrique du nord trouvées en Gaule, (Supplément à Gallia 36), Paris, 1978.

FleISCHER 2007 : F. FleISCHER, Siedlungsarchäologie auf dem Mont Beuvray. Die Ausgrabungen der Universitäten Kiel und Leipzig 1989-1998 im Oppidum Bibracte (Nièvre - Saône-et-Loire), thèse de doctorat inédite, Universität Leipzig, 2007.

FRANCE 2001 : J. FRANCE, Quadragesima galliarum : l'organisation douanière des provinces alpestres, gauloises et germaniques de l'Empire romain :

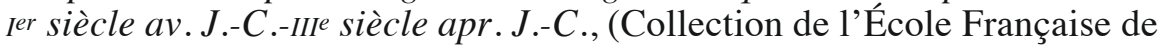
Rome 278), Rome, 2001.

Frey-Kupper, Stannard 2010 : S. Frey-Kupper, Cl. Stannard, Les imitations pseudo-Ebusus/Massalia en Italie centrale : typologie et structure, présence dans les collections et les trouvailles de France, $R N, 2010$, p. 109-147.

García-Bellido 2004 : M.-P. García-Bellido, Las legiones hispánicas en Germania. Moneda y ejército, (Anejos de Gladius 6), Madrid, 2004.

GENECHESI 2012 : J. GENECHESI, Entre Marseille et Rome, le rôle de la monnaie dans le développement économique de l'axe rhodanien du IIe siècle av. J.-C. au rer siècle apr. J.-C., thèse de doctorat inédite, Université Paris I PanthéonSorbonne, 2012.

Guilhot, Goy (dir.) 1992 : J.-O. Guinloy, C. Goy (dir.), $20000 \mathrm{~m}^{3}$ d'histoire : les fouilles du parking de la Mairie à Besançon (23 mai-5 oct. 1992, Musée des Beaux-arts et d'archéologie, Besançon), Besançon, 1992.

Gruel 2005 : K. Gruel, L'alignement du denier gaulois sur l'étalon romain : datation et impact économique, dans Die Kelten und Rom: neuе numismatische Forschungen. Les Celtes et Rome : nouvelles études numismatiques, Fond de Gras-Titelberg, Luxemburg, 30.4.-3.5.1998, J. Metzler, D. Wigg-Wolf (eds.), (Studien zu Fundmünzen der Antike 19), Mayence, 2005, p. 29-37.

Gruel, Popovitch 2007 : K. Gruel, L. Popovitch, Les monnaies gauloises et romaines de l'oppidum de Bibracte, (Bibracte 13), Glux-en-Glenne, 2007.

Gruel et al. 2009 : K. Gruel, Ph. Charnotet, M. Troubady, Comparaison des faciès monétaires d'Orléans (Loiret), Levroux (Indre) et Châteaumeillant (Cher), dans L'âge du fer dans la boucle de la Loire. Les Gaulois sont dans la ville. Actes du XXXII colloque de l'Association Française pour l'Étude de l'Âge du Fer, Bourges, $1^{\text {er }} 4$ mai 2008, O. Buchsenschutz, M.-B. Chardenoux, S. Krausz (éd.), (Revue archéologique du Centre de la France. Supplément 35), Tours, 2009, p. 121-134.

Hiernard 1982 : J. Hiernard, Corbilo et la route de l'étain, Bulletin de la Société des Antiquaires de l'Ouest, 16, 1982, p. 497-578.

KeMmers 2006 : Fl. KeMmers, Coins for a legion: an analysis of the coin finds of the Augustan legionary fortress and Flavian canabae legionis at Nijmegen, (Studien zu Fundmünzen der Antike 21), Mayence, 2006.

KeMmers 2008 : Fl. Kemmers, Contexts and phases: suggestions for a new approach to Celtic coins in Roman forts, dans Coinage in the iron age: essays in honour of Simone Scheers, J. van Heesch, I. Heeren (eds.), London, 2009, p. 271-278. 
KÜHLBORN 1992 : J.-S. KüHLBORn, Das Römerlager in Oberaden III: die Ausgrabungen im nordwestlichen Lagerbereich und weitere Baustellenuntersuchungen der Jahre 1962-1988, (Bodenaltertümer Westfalens 27), Münster, 1992.

Kubon 2011 : R. Kubon, Forschungen zum römischen Höchst, Stadt Frankfurt am Main. Katalog der Fundstellen in Frankfurt-Höchst und Umgebung, (Schriften des Archaölogischen Museums Frankfurt 23/1), Francfort sur le Main, 2011.

LАMвOT 2004 : B. LAMBOT, Les monnaies gauloises en or de Ribemont-sur-Ancre (Somme). Réflexion sur leur datation, Revue Archéologique de Picardie, 1/1, 2004, p. 123-138.

Lambot, Casagrande 1997 : B. Lambot, P. Casagrande, Une monnaie d'Ebusus sur l'oppidum de «Vieux-Laon» à Saint-Thomas (Aisne). Nouvelles données archéologiques sur Bibrax et le camp de Mauchamp (César B.G. II), Bulletin de la Société Archéologique Champenoise, 90, 1997, no 2, p. 15-29.

Lardy et al. 1987 : J.-M. Lardy, H. Meyer, V. Rebour, N. VanPeEne, Monnaies celtiques en situation stratigraphique sur le site d'Epiais-Rhus (Val d'Oise), dans Monnaies gauloises découvertes en fouille, J.-L. Brunaux, K. Gruel (dir.), (Dossier de Protohistoire 1), Paris, 1987, p. 152-210.

Luginbühl, Schneiter 1999 : Th. Luginbühl, A. Schneiter, La fouille de Vidy «Chavannes 11 », 1989-1990: Trois siècles d'histoire à Lousonna, Le mobilier archéologique, (Cahiers d'Archéologie Romande 74), Lausanne, 1999.

LulEy 2008 : B.P. Luley, Coinage at Lattara. Using Archaeological Context to Understand Ancient Coins, Archaeological Dialogues, 15/2, 2008, p. 174-195.

MANGARD 2008 : M. MANGARD, Le sanctuaire gallo-romain du Bois l'Abbé à Eu, Seine-Maritime, (Revue du Nord. Hors série. Collection Art et Archéologie 12), Villeneuve-d'Ascq, 2008.

Mantel (éd.) 1998 : E. Mantel (éd.), Le sanctuaire de Fesques «Le Mont du Val aux Moines », Seine Maritime, (Nord-Ouest Archéologie 8), Berck-sur-Mer, 1998.

MAZARD 1955 : J. MAZARD, Corpus nummorum Numidiae Mauretaniaeque, Paris, 1955.

Martin 2011 : St. Martin, Monnaies gauloises, usagers romains, et vice versa. L'exemple de la Gaule de l'est, dans Aspects de la romanisation dans l'Est de la Gaule, M. Reddé et al. (éd.), (Bibracte 21/2), Glux-en-Glenne, 2011, p. 937-944.

MetZler 1995 : J. Metzler, Das treverische Oppidum auf dem Titelberg: G.-H. Luxemburg : Zur Kontinuität zwischen der spätkeltischen und der frührömischen Zeit in Nord-Gallien, (Dossiers d'Archéologie du MNHA 3), Luxembourg, 1995.

Milcent 2006 : P.-Y. Milcent, Examen critique des importations méditerranéennes en Gaule centrale et occidentale les attributions douteuses, erronées ou falsifiées, dans De la Méditerranée vers l'Atlantique. Aspects des relations entre la Méditerranée et la Gaule centrale et occidentale (VIII-IIIe s. av.J.-C.), D. Frère (dir.), (Archéologie et culture), Rennes, 2006, p. 117-133. 
Morscheiser-Niebergall 2009 : J. Morscheiser-Niebergall, Die Anfänge Triers im Kontext augusteischer Urbanisierungspolitik nördlich der Alpen, (Philippika 30), Wiesbaden, 2009.

Nickel et al. 2008 : Cl. Nickel, M. Thoma, D. WigG-Wolf, Martberg. Heiligtum und Oppidum der Treverer I. Der Kultbezirk. Die Grabungen 1994-2004, (Berichte zur Archäologie an Mittelrhein und Mosel 14), Koblenz, 2008.

PION 1996 : P. PIon, Les habitats laténiens tardifs dans la vallée de l'Aisne : contribution à la périodisation de la fin du second Âge du Fer en Gaule nordorientale : la Tène C2 - période augustéenne précoce, II -Ier S. av.J.-C., thèse de doctorat inédite, Université Paris I Panthéon-Sorbonne, 1996.

Piton, Dilly 1985 : D. Piton, G. Dilly, Le fanum des «Châtelets » de VendeuilCaply (Oise), Revue archéologique de Picardie, 1/1, 1985, p. 25-47.

Pommeret (éd.) 2001 : C. Pommeret (éd.), Le sanctuaire antique des Bolards à Nuit-Saint-Georges, Côte-d'Or, (Revue Archéologique de l'Est, Supplément 16), Dijon, 2001.

Poux 2004 : M. Poux, L’âge du vin. Rites de boisson, festins et libations en Gaule indépendante, (Protohistoire européenne 8), Montagnac, 2004.

Poux, Savay-Guerraz 2003 : M. Poux, H. SAvay-Guerraz (dir.), Lyon avant Lugdunum. Exposition, Musée de la civilisation gallo-romaine de Lyon, marsnovembre 2003, Lyon, 2003.

Reddé, Schnurbein 2001 : M. Reddé, S. von Schnurbein (dir.), Alésia. Fouilles et recherches franco-allemandes sur les travaux militaires romains autour du Mont-Auxois (1991-1997), (Mémoires de 1'Académie des Inscriptions et Belles Lettres 22), Paris, 2001.

Rossi (dir.) 2005 : Fr. Rossi (dir.), Théâtre d'Alésia. Rapport d'activité 2005 , rapport de fouille, Archeodunum SA, 2005, consulté le 12/11/2011 sur http://www .archeodunum.ch.

Santrot, Aubin 2002 : J. Santrot, G. Aubin, Les monnaies « exotiques » de Darun, en Nivillac (Morbihan), Revue archéologique de l'Ouest, 19, 2002, p. 219-229.

SéGuier, Mallet 2005 : J.-M. SÉGuier, Fr. Mallet et al., Le faciès augustéen de la plaine de France d'après le mobilier des établissements de Charny et de Compas (Seine-et-Marne), dans SFECAG, Actes du congrès de Blois, L. Rivet (éd.), Marseille, 2005, p. 529-560.

STANnARd à paraître : Cl. STANnARD, Are Ebusan and pseudo-Ebusan coin at Pompeii a sign of intensive contacts with the Island of Ebusus?, dans Actas de la Reunión científica, Ebusus y Pompeya. Testimonios monetales de una relación (Roma, 12-13 Novembre 2010, Escuela Española de Historia y Arqueología en Roma, CSIC), A. Arévalo González, D. Bernal Casasola, D. Cottica (eds.), Cadix, à paraître.

Toledo i Mur 1997-1998 : A. Toledo i Mur, La Croix du Buis (Arnac-la-Poste, Haute-Vienne). Un entrepôt du rer siècle a.C., Aquitania, 15, 1997-1998, p. 109-140. 
Trébuchet et al. 2009 : E. Trebuchet et al., Une unité d'exploitation rurale de la première moitié du IIIe s. av. J.-C. à Mer (Loir-et-Cher), dans L'âge du fer dans la boucle de la Loire. Les Gaulois sont dans la ville. Actes du XXXII colloque de l'Association Française pour l'Étude de l'Âge du Fer, Bourges, $1^{\text {er-4 }} 4$ mai 2008, O. Buchsenschutz, M.-B. Chardenoux, S. Krausz (éd.), (Revue archéologique du Centre de la France. Supplément 35), Tours, 2009, p. 157-165.

Vaussanvin 1987 : H. VAussanvin, Le site des Sept Fontaines à Tournus, dans Monnaies gauloises découvertes en fouille, J.-L. Brunaux, K. Gruel (dir.), (Dossier de Protohistoire 1), Paris, 1987, p. 236-254.

VERGER 2011 : St. VERGER, Du recyclage à la contrefaçon. Histoires de vases métalliques de l'Âge du Fer, dans L'Âge du Faux : l'authenticité en archéologie, M.-A. Kaeser (dir.), Hauterive, 2011, p. 136-145.

WeILLER 1977 : R. WeILler, Die Münzfunde aus der Grabung vom Tetelbierg, Publications de la section historique de l'Institut grand-ducal de Luxembourg, 91, 1977, p. 117-199. 